\title{
编写分析化学教材的思考与实践
}

陈永雷, 何疆, 陈宏丽, 陈兴国 ${ }^{*}$

兰州大学化学化工学院, 兰州 730000

摘要: 分析化学教材是本科生学习分析化学、激发创新热情、培养创新思维、提升创新能力的重要载体。编写符合这些 要求的分析化学教材已成为分析化学教育工作者必须深入思考、着力解决的重要问题。鉴于此, 本文介绍了笔者在编写 分析化学教材过程中的一些思考和做法以及所编写的《分析化学》教材的特色, 希望能起到抛砖引玉的作用。

关键词: 分析化学; 教材; 特色

中图分类号: G64; O6

\section{Thinking and Practice of Compiling Analytical Chemistry Textbook}

Yonglei Chen, Jiang He, Hongli Chen, Xingguo Chen *

College of Chemistry and Chemical Engineering, Lanzhou University, Lanzhou 730000, China.

\begin{abstract}
An analytical chemistry textbook is an important carrier for undergraduates to study analytical chemistry, stimulate innovation enthusiasm, cultivate innovative thinking and enhance innovation ability. Therefore, compiling of analytical chemistry textbooks that meet these requirements has become an important issue for analytical chemistry educators to think deeply and work on. In view of this, this paper introduced the authors' thinking and practice in the process of compiling an analytical chemistry textbook and the characteristics of the textbook. The authors hope to offer a good starting point for the analytical chemistry teaching peers.
\end{abstract}

Key Words: Analytical chemistry; Textbook; Characteristics

分析化学教材是本科生学习分析化学、激发创新热情、培养创新思维、提升创新能力的重要载 体, 编写内容科学、时代性强并具于一定前瞻性和广泛适用性的分析化学教材不仅对本科分析化学 教学、分析化学创新性人才的培养具有十分重要的意义, 也是分析化学教育工作者应该深入思考、 着力解决的重要问题之一。鉴于此, 在认真研读国内外现行分析化学教材的基础上 ${ }^{[1-5]}$, 根据教育部 化学与化工学科教学指导委员会制订的关于化学、应用化学、材料化学以及药学、环境科学等专业 化学教学基本内容, 结合在完成教育部 “国家理科基地创建分析化学名牌课程” 和 “甘肃省名牌课 程建设” 项目过程中对分析化学课程体系、教学内容及教学方法的改革和实践, 笔者编写了《分析 化学》 (第一版) 教材 ${ }^{[6]}$ 。该教材于 2012 年由高等教育出版社出版并在兰州大学等高校使用。根据使用 情况和学生及同行的意见和建议, 笔者对第一版教材的内容进行了修改和补充, 形成了《分析化学》 (第二版)教材 ${ }^{[7]}$, 该教材于 2021 年由高等教育出版社出版。现将笔者在编写该教材过程中的一些思考 和做法以及所编写的《分析化学》教材的特色介绍给大家, 希望能起到抛砖引玉的作用, 促进分析

收稿: 2021-06-21; 录用: 2021-08-11; 网络发表: 2021-08-19

“通讯作者, Email: chenxg@1zu.edu.cn

基金资助: 国家自然科学基金(20875040，21375053) 
化学教材编写的蓬勃发展。

\section{1 科学性}

分析化学教材是本科生学习分析化学基本内容和培养创新能力的主要载体, 为了使本科生通过 学习《分析化学》教材的内容, 达到启迪创新思维、培养创新能力的作用, 教材内容的科学性是第 一位的。笔者认为, 教材内容的科学性主要体现在教材不仅要包括分析化学最主要的理论、原理、 方法、技术和应用, 而且要反映分析化学的发展历程、发展趋势、科学思维方法、分析和解决科学 问题的途径等内容。为此, 我们在编写分析化学教材时力求全面展现分析化学的基础理论、清晰阐 述分析化学解决主要问题的思路、详细说明一些重要计算公式的推导及内在规律等。主要包括: (1) 传 统的分析化学教材在介绍一元线性回归过程时很少介绍一元线性回归方程 $y=a+b x$ 中 $y 、 a 、 b$ 的标 准偏差 $s_{y} 、 s_{a} 、 s_{b}$ 以及根据回归方程计算得到的分析结果 $c$ 的标准偏差 $s_{c}$, 这样不仅有可能使学生认为 $y 、 a 、 b$ 和 $c$ 没有误差, 而且无法满足科学研究的要求。为解决这一问题, 我们在教材中介绍了计算 $s_{y} 、 s_{a} 、 s_{b}$ 和 $s_{c}$ 的计算公式并给出了具体例题 ${ }^{6,7]}$ 。(2) 在介绍酸碱溶液中各型体分布分数的计算公式 之后, 明确指出 “仔细观察上述弱酸各型体分布分数的表达式可发现这样的规律: a) 各型体分布分 数的分母按 $\left[\mathrm{H}^{+}\right]$降幂排列, 第一项为 $\left[\mathrm{H}^{+}\right]^{n}$ ( $n$ 为弱酸的元数), 最后一项为弱酸各解离常数的乘积; 某 项的 $\left[\mathrm{H}^{+}\right]$幂次降低 1 , 就增加一相应的 $K_{\mathrm{a}_{i}}$ 并与其相乘; b) 分母中的第一项为 $\delta_{\mathrm{H}_{n} \mathrm{~A}}$ 的分子, 第二项为 $\delta_{\mathrm{H}_{n-1} \mathrm{~A}^{\mathrm{A}}}$ 的分子, 余类推。” 然后进一步指出应用分布分数不仅可根据酸碱溶液的分析浓度求得溶液中 溶质各种型体的平衡浓度, 还可以据此选取实验的适宜酸度 ${ }^{[6,7]}$ 。这样编写不仅能使学生深刻掌握分 布分数计算公式的本质和规律, 而且能降低学生记忆公式的压力, 还可以培养学生的创新思维能力; （3）虽然通过计算机技术可以精确计算各种酸碱溶液的 $\mathrm{pH}$, 但这样处理弱化了分析化学解决这类问 题的思路和方法, 不利于培养学生的科学思维能力和创新能力。为此, 笔者在介绍各种酸碱溶液 $\mathrm{pH}$ 的计算时, 坚持从酸碱质子理论出发, 依据所考查溶液的质子条件结合酸(碱)的离解常数和其他平 衡常数、酸(碱)的浓度推导出精确式, 然后再依据具体条件和要求通过合理近似得到相应的近似 式 ${ }^{[6,7]}$ 。这种编写方法如实呈现了分析化学解决具体问题的思路的科学性和严谨性, 对培养学生的逻 辑思维能力和解决科学问题的能力是十分有利的; (4) 在介绍酸溶液的 $\mathrm{pH}$ 计算方法之后, 指出碱溶 液 $\mathrm{pH}$ 的计算与相应酸溶液 $\mathrm{pH}$ 的计算公式十分相似, 仅需将相应公式中的 $\left[\mathrm{H}^{+}\right]$和 $K_{\mathrm{a}}$ 替换为 $\left[\mathrm{OH}^{-}\right]$和 $K_{\mathrm{b}}$ 即可。这种编写方法不仅能让学生了解酸碱溶液 $\mathrm{pH}$ 的计算在本质上是一致的, 还为学生介绍了处理 相似问题的简洁方式 ${ }^{[6,7]}$; (5) 在介绍影响沉淀溶解度的因素时, 为使学生深刻全面了解同离子效应、 盐效应、酸效应和络合效应等对微溶化合物溶解度 $(s)$ 的影响和方便学生定量计算具体情况下微溶化 合物的 $s$, 我们将计算 $\mathrm{M}_{m} \mathrm{~A}_{n}$ 型微溶化合物 $s$ 的通式引入教材 ${ }^{[6,7], ~}$ 如下所示:

$$
\begin{aligned}
& \lg s=\frac{1}{m}\left(\lg K_{\mathrm{sp}}^{0}+m \lg \alpha_{\mathrm{M}}+n \lg \alpha_{\mathrm{A}(\mathrm{H})}-m \lg \gamma_{\mathrm{M}}-n \lg \gamma_{\mathrm{A}}-n \lg c_{\mathrm{A}}-m \lg m\right) \\
& \text { 络合效应 酸效应盐效应同离子效应 } \\
& \lg s=\frac{1}{n}\left(\lg K_{\mathrm{sp}}^{0}+m \lg \alpha_{\mathrm{M}}+n \lg \alpha_{\mathrm{A}(\mathrm{H})}-m \lg \gamma_{\mathrm{M}}-n \lg \gamma_{\mathrm{A}}-m \lg c_{\mathrm{M}}-n \lg n\right) \\
& \text { 络合效应 酸效应盐效应同离子效应 } \\
& \lg s=\frac{1}{m+n}\left(\lg K_{\mathrm{sp}}^{0}+m \lg \alpha_{\mathrm{M}}+n \lg \alpha_{\mathrm{A}(\mathrm{H})}-m \lg \gamma_{\mathrm{M}}-n \lg \gamma_{\mathrm{A}}-m \lg m-n \lg n\right) \\
& \text { 络合效应 酸效应盐效应 }
\end{aligned}
$$

通过上述公式学生不仅能快速准确地计算各种情况下 $\mathrm{M}_{m} \mathrm{~A}_{n}$ 型微溶化合物的 $s$, 而且能深刻全面 了解各种因素对 $s$ 的影响程度并根据具体情况通过控制主要因素调控微溶化合物的 $s$ 。 


\section{2 时代性}

同其他学科的教材一样, 分析化学教材同样具有鲜明的时代特征, 即它不仅要满足新时代和立 德树人的要求, 还必须反映分析化学学科的最新理论和应用。鉴于此, 笔者在编写《分析化学》教 材时, 充分考虑了新时代和立德树人的要求, 对其内容和表现方式进行了重组和改革。主要包括在 绪论中通过二维码向学生展示了分析化学发展目标(即 Selectivity, Sensitivity, Speediness, Accuracy, Automatization, Application, $3 \mathrm{~S}+3 \mathrm{~A}$ ), 引导学生为实现该目标进行创新性的研究并努力取得具有原 始创新性的成果, 为分析化学的发展和中国特色社会主义的建设贡献自己的力量; 在氧化还原滴定 法中通过二维码介绍了 “水质高锰酸盐指数的测定” 和 “水质化学需氧量的测定” 的国家标准 GB11892-89和GB11914-89 [7]。通过这两个国家标准的介绍, 使学生体会到我们国家对环境保护的 重视，激发学生深刻理解习近平总书记的 “绿水青山就是金山银山” 的内涵并为之努力奋斗。

分析化学教材作为本科生学习分析化学基本内容和培养创新能力的主要载体, 为了使其真正起 到启迪学生创新思维、培养学生创新能力的作用, 笔者在编写《分析化学》教材时, 根据近几十年 来分析化学在理论和应用研究方面所取得的主要进展和成果, 精选了最具代表性的内容并将其编入 分析化学教材, 力求使该教材具有鲜明的时代特征。主要包括: (1) 计算酸碱滴定、氧化还原滴定、 沉淀滴定终点误差的林邦公式 ${ }^{[6,7]}$; (2) 纳米粒子作为指示剂在滴定钙离子中的应用 ${ }^{[7]}$; (3) 毛细管电 泳及相关联用技术 ${ }^{[6,7]}$ 。教学实践表明, 将上述内容引入教材, 不仅能使学生及时了解分析化学学科 的最新成果, 而且拓宽了学生的眼界, 激发了学生学习分析化学的兴趣, 培养了学生的科学思维方 法, 提升了学生的创新能力。

除了在教材内容上反映分析化学的时代特征, 在编写教材时我们注意将新的内容展现方式引入 教材, 以拓宽教材的内涵。为此, 我们在编写《分析化学》(第二版)时, 将分析化学的最新应用和发 展前沿如分析化学发展目标 $(3 \mathrm{~S}+3 \mathrm{~A})$ 、人工神经网络简介、纳米粒子在滴定钻离子中的应用、氮掺 杂碳量子点的固相合成及其在细胞内 $\mathrm{Fe}^{3+}$ 成像分析中的应用、基于 $\mathrm{Fe}_{3} \mathrm{O}_{4}$ 纳米颗粒检测乳制品中三聚 氧胺、单滴微萃取应用简介、固相微萃取联用技术、二维毛细管电泳、毛细管电泳联用技术等通过 数字化资源的方式予以呈现 ${ }^{[7]}$, 不仅丰富了教材内容, 而且为学生学习这些内容提供了时间和空间 上的便利。

\section{3 前瞻性}

为了培养学生的创新能力, 分析化学教材还应具有一定的前瞻性。为此, 在编写《分析化学》 时, 我们结合分析化学的基本内容适时地对分析化学刚刚出现的、将来有可能成为分析化学的主要 理论或应用进行了简要介绍, 以引起学生的关注并进行有关的探索。例如在介绍完分析化学中的误 差和数据处理的主要内容后, 对化学信息学的定义、主要研究内容、互联网上的化学信息学工具和 杂志进行了简要介绍 ${ }^{[6,7]}$, 这些内容不仅对学生了解化学信息学的主要内容及其在化学尤其是分析化 学中的应用具有重要意义, 而且对学生了解在信息时代分析化学的发展方向和趋势也是十分必要的。 在介绍金属离子指示剂时, 适时介绍了纳米材料指示剂在络合滴定中的应用及该指示剂的特点, 希 望能引导学生结合分析化学基本内容进行深入的思考并在创新创业中开展相关研究。此外, 考虑到 纳米科技已经并必将对分析化学的发展起到重大的促进作用, 笔者在编写 《分析化学》时分别在相 关章节介绍了纳米材料的主要化学制备方法、纳米材料在吸光光度法中的应用、纳米探针的制备及 应用等内容 ${ }^{[6,7]}$, 希望能起到开拓学生视野、培养学生创新能力的作用。

\section{4 适用性}

我们认为一本优秀的教材除了具有科学性、时代性、前瞻性之外, 还应该具有广泛的适用性, 只有这样, 才能具有强大的生命力并起到应有的作用。因此, 我们在编写《分析化学》时, 除了努 力使该教材重点突出、层次分明、遵从循序渐进的教学规律外, 还特别注意使其具有尽可能广泛的 
适用性。为此, 在保证分析化学基本内容不受削弱的前提下, 适当介绍了分析化学对医学、生命科 学、环境科学、食品科学等领域的重要性和在这些领域的典型应用, 主要内容包括缓冲溶液在调节 人体体液 $\mathrm{pH}$ 中的作用、水质高锰酸盐指数的测定、水质化学需氧量的测定、氮掺杂碳量子点的固相 制备及其在细胞内 $\mathrm{Fe}^{3+}$ 成像分析中的应用、基于 $\mathrm{Fe}_{3} \mathrm{O}_{4}$ 纳米颗粒检测乳制品中三聚氧胺、单滴微萃取 应用简介、固相微萃取联用技术等, 使该教材不仅可作为综合性大学和高等师范院校化学化工类专 业本科生分析化学课程的教材, 也可作为从事分析化学研究或相关工作的人员的参考书籍 ${ }^{[6,7] 。}$

\section{5 结语}

本文介绍了笔者在编写分析化学教材过程中围绕新时代和立德树人的要求及培养本科生创新 能力的目的所进行的一些思考和做法。笔者认为, 在以后的教材编写中, 应努力克服现有分析化学 教材重视知识的整合梳理、知识体系的构建, 但缺乏对学生独立思考的引领和探索精神激发的不足, 通过对分析化学理论、应用和其所包含的科学内涵进行深入的研究, 才能编写出具有科学性、时代 性、前瞻性和适用性的分析化学教材。希望笔者的思考、做法和建议能引起大家的关注和共勉, 促 进分析化学教材编写和分析化学教学工作的发展, 加速分析化学创新性人才的培养。

\section{参 考 文 献}

[1] 武汉大学. 分析化学. 第3版. 北京: 高等教育出版社, 1995.

[2] 武汉大学. 分析化学. 第4版. 北京: 高等教育出版社, 2000.

[3] 武汉大学. 分析化学(上册). 第5版. 北京: 高等教育出版社, 2006.

[4] 李龙泉, 林长山, 朱玉瑞, 吕敬慈, 江万权. 定量化学分析. 合肥: 中国科技大学出版社, 2002.

[5] 彭崇慧, 冯建章, 张锡瑜. 定量化学分析简明教程. 第2版. 北京: 高等教育出版社, 1997.

[6] 陈兴国, 何疆, 陈宏丽, 陈永雷. 分析化学. 第1版. 北京: 高等教育出版社, 2012.

[7] 陈兴国, 何疆, 陈宏丽, 陈永雷. 分析化学. 第2版. 北京: 高等教育出版社, 2021. 